\title{
Skin glucose metabolism and microvascular blood flow during local insulin delivery and after an oral glucose load
}

Fredrik Iredahl, Alexandra Högstedt, Joakim Henricson, Folke Sjöberg, Erik Tesselaar and

Simon Farnebo

\section{Journal Article}

\section{Tweet}

N.B.: When citing this work, cite the original article.

Original Publication:

Fredrik Iredahl, Alexandra Högstedt, Joakim Henricson, Folke Sjöberg, Erik Tesselaar and Simon Farnebo, Skin glucose metabolism and microvascular blood flow during local insulin delivery and after an oral glucose load, Microcirculation, 2016. 23(7), pp.597-605.

http://dx.doi.org/10.1111/micc.12325

Copyright: Informa Healthcare / Wiley: 12 months

http://eu.wiley.com/WileyCDA/

Postprint available at: Linköping University Electronic Press

http://urn.kb.se/resolve?urn=urn:nbn:se:liu:diva-132368

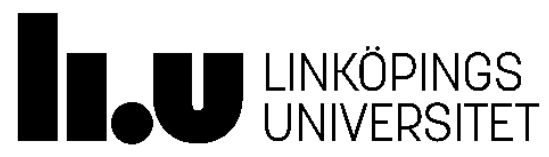




\section{Skin glucose metabolism and microvascular blood flow during local insulin delivery and after an oral glucose load}

Fredrik Iredahl ${ }^{1}$, Alexandra Högstedt ${ }^{1}$, Joakim Henricson ${ }^{2}$, Folke Sjöberg ${ }^{1,3}$, Erik Tesselaar ${ }^{1,4}$, Simon Farnebo ${ }^{3}$

1. Department of Clinical and Experimental Medicine, Linköping University, Linköping, Sweden

2. Department of Dermatology and Venerology, Department of Clinical and Experimental Medicine Linköping University, Linköping, Sweden

3. Department of Hand Surgery, Plastic Surgery, and Burns, Linköping University, Linköping, Sweden

4. Department of Radiation Physics, Linköping University, Linköping, Sweden

\section{Corresponding author}

Fredrik Iredahl, M.D.

Department of Clinical and Experimental Medicine

Faculty of Health Sciences

Linköping University

SE-58185 Linköping, Sweden

E-mail: fredrik.iredahl@liu.se

Running head: Metabolism and microvascular effects of insulin in skin

\section{Contribution to study:}

Conceived and designed the experiments: FI, AH, JH, FS, ET, SF. Performed the experiments: FI, AH, JH. Analyzed/interpreted the data: FI, AH, JH, FS, ET, SF. Wrote the paper: FI, AH, JH, FS, ET, SF.

\section{Grants:}

The study has been financially supported by ALF grants, Region Östergötland, by Sinnescentrum, Region Östergötland, and by The Grönberg Foundation.

\section{Keywords:}

Insulin, Skin, Metabolism, Microcirculation, Microdialysis 


\section{Abstract}

Insulin causes capillary recruitment in muscle and adipose tissue, but the metabolic and microvascular effects of insulin in the skin have not been studied in detail. The aim of this study was to measure glucose metabolism and microvascular blood flow in the skin during local insulin delivery and after an oral glucose load using intracutaneous microdialysis and laser speckle contrast imaging (LSCI). Within 15 minutes of local insulin delivery, microvascular blood flow in the skin increased (urea clearance: $\mathrm{p}=0.047$, LSCI: $\mathrm{p}=0.002)$ paralleled by increases in pyruvate $(\mathrm{p}=0.01)$ and lactate $(\mathrm{p}=0.04)$, indicating an increase in glucose uptake. Thus, local delivery of insulin to the skin via microdialysis resulted in rapid vasodilatation, paralleled by an increased glucose uptake. An oral glucose load increased urea clearance from the catheters, indicating an increase in skin perfusion, although no perfusion changes were detected with LSCI. The time course of the effects of insulin on glucose uptake in the skin differed markedly depending on whether insulin is delivered directly to the interstitium via microdialysis, or via a systemic route. These results show that insulin has specific metabolic and vasodilatory effects in the skin both when given locally and after systemic delivery through an oral glucose load.

\section{Introduction}

Insulin has, besides its important role as regulator of blood glucose levels in the body, vasoactive properties. It has been shown that insulin causes tissue capillary recruitment to increase the endothelial surface available for the transportation of insulin, glucose, and other nutrients to the interstitium during a hyperinsulinemic euglycemic clamp [22], after a mixed meal [38] and during local administration of insulin [34]. A disrupted interplay between the metabolic and vasoactive functions of insulin has been proposed to play a key role in the development of microcirculatory dysfunction and insulin resistance [5,17]. When insulin fails to increase tissue blood flow, both its 
own delivery as well as the supply of glucose to the surrounding tissue is reduced [2,6]. This phenomenon can be seen in hypertension [33] and obesity [7,17,20]. Research that aims to better understand the relation between the metabolic and vasoactive actions of insulin in the body are therefore of clinical interest.

It has been shown that systemically induced hyperinsulinemia augments capillary recruitment in the skin, and that this is paralleled by insulin-mediated microvascular recruitment in muscle [22]. Also, it has been shown that decreased insulin sensitivity is associated with an impaired capillary recruitment and impaired acetylcholine-mediated vasodilatation in the skin [7]. The microcirculation of the skin is in various other contexts considered to be representative for systemic microvascular function [12].

To study the vascular effects of insulin in the skin, it can be delivered locally or systemically. Transdermal iontophoresis has been used for local delivery of insulin to the skin, in combination with laser Doppler flowmetry for measurement of microvascular responses in the skin [14,23,29,30,34]. However, the delivery of insulin via iontophoresis has been found challenging, because of a substantial nonspecific vasodilatation caused by the diluting medium of the drugs that have been used [14,23,34]. Also, because metabolic effects cannot be directly studied using optical techniques, previous studies using transdermal iontophoresis have been limited to measurement of vascular effects.

Intracutaneous microdialysis, on the other hand, makes it possible to simultaneously deliver substances to the skin and measure changes in the concentration of metabolites [10] and blood flow $[9,11]$ in the same tissue volume. Microdialysis has previously been used to study the metabolic and vascular effects of insulin in adipose tissue and skeletal muscle, by monitoring changes in tissue metabolites and blood flow after systemic hyperinsulinemia [28], as well as after local administration of insulin through the catheter into the tissue [27]. However, the effects of systemic hyperinsulinemia and local administration of insulin on skin microcirculation and metabolism have not been studied in detail using microdialysis.

The aim of this study was therefore to study the metabolic and vascular actions of insulin in the skin, when it is (1) endogenously produced and delivered to the skin via the bloodstream or (2) 
directly delivered to the interstitium via a microdialysis catheter. We hypothesized that insulin would cause a change in the concentration of metabolic markers, glucose, lactate and pyruvate, in the skin surrounding the catheter, and that insulin would cause a local increase in blood flow. We also hypothesized that the metabolic and vascular effects of insulin would differ depending on whether it would (1) be endogenously delivered to the tissue via the microvasculature after an oral glucose load, or (2) delivered directly through the microdialysis catheter.

\section{Method}

\section{Subjects}

Fourteen healthy subjects (7 female) participated in the study (Table 1). Their mean age was $23 \pm 5.1$ years (range 19-40). They were non-smokers and used no regular medication except for oral contraceptives. The subjects arrived in the morning after an overnight fast and were only allowed to drink water during the experiments. All subjects gave their written informed consent before their participation. The study was performed according to the Helsinki declaration and was approved by the regional ethical committee of Linköping (application ID: 2011/362/31).

\section{Study protocol}

The skin of the volar aspect of the forearm was disinfected (Chlorhexidine, Fresenius Kabi AB, Uppsala, Sweden). Then, a $0.1 \mathrm{ml}$ Xylocaine injection (Xylocaine $20 \mathrm{mg} / \mathrm{ml}$; AstraZeneca, Södertälje, Sweden) was given at two sites, at approximately $3 \mathrm{~cm}$ distance from each other. A microdialysis catheter (CMA 71, M dialysis AB, Stockholm, Sweden) was inserted at each of the two sites, as follows. An 18-Gauge cannula (BD Venflon ${ }^{\mathrm{TM}}$ Pro, Bection Dickinson Infusion Therapy AB, Helsingborg, Sweden) was pushed through the surface of the skin and advanced along the skin surface at a depth of approximately $1 \mathrm{~mm}$. The membrane of the catheters were located at least $2.5 \mathrm{~cm}$ from the xylocaine injection site. We have previously confirmed the depth of the catheters using a 
DermaScan ultrasound device to be approximately $0.8 \mathrm{~mm}$, on the boundary of the dermis and hypodermis [31]. The steel mandrin was then removed and the cannula cut off, so that only the plastic cannula was left inside the skin. Thereafter, the microdialysis catheter was inserted into the plastic cannula, after which the plastic cannula was removed. Finally, thin transparent adhesive film (Tegaderm, 3M Healthcare, St. Paul, USA) was attached over the insertion point to keep the catheters in place. The two catheters were placed in parallel and each catheter was connected to a microinjection pump (CMA 107, CMA AB, Solna, Sweden), and perfused with a perfusate containing 5:6 Ringer-Acetate with addition of $2.5 \%$ albumin, $30 \mathrm{mM}$ urea (APL Apoteket, Umeå, Sweden) and 1:6 insulin diluting medium (Insulin Diluting Medium for Novorapid and Levemir, Novo Nordisk A/S, Bagsværd, Denmark), resulting in a final concentration of $2.1 \%$ albumin and $25 \mathrm{mM}$ urea. To minimize adsorption of insulin by the catheter material, the catheters were perfused for 90 minutes before insertion in the skin.

An outline of the complete protocol is presented in Figure 1. After the insertion, the catheters were perfused for 90 minutes to allow for the tissue to recovery from the insertion trauma and to verify adequate function of the catheters. Then, a 60-minute baseline period was observed. After that, both pumps were opened and one of the syringes (106 Syringe, M dialysis AB, Stockholm, Sweden) containing the perfusion fluid was exchanged for a syringe containing 5:6 Ringer-Acetate with addition of $30 \mathrm{mM}$ urea and $2.5 \%$ albumin and 1:6 of $100 \mathrm{U} / \mathrm{mL}$ insulin aspart (Novorapid 100 U/mL, Novo Nordisk A/S, Bagsværd, Denmark). The final concentration of insulin aspart in insulin catheter was $16.7 \mathrm{U} / \mathrm{mL}$ The other syringe continued with the original solution throughout the protocol. When opening and closing the pumps, an automatic flush sequence is initiated, which is why the first sample after the exchange of syringes was discarded. The responses to insulin addition in insulin catheter and to control substance in the control catheter were studied for 60 minutes. Thereafter a WHO standard of oral glucose tolerance test (OGTT) was performed. 75 g glucose (Apotek Produktion \& Laboratorier AB, Stockholm, Sweden) diluted in $2 \mathrm{dl}$ water was ingested within 5 minutes. 
In 6 subjects, no insulin was added to the perfusate of the microdialysis catheter, and no glucose was given orally (Table 1, Figure 1), while the protocol was otherwise identical. These subjects were used as controls to investigate any effects not related to local or systemic insulin.

All microvials were labeled and then weighed (CPA225D, Sartorius Weighing Technology GmbH, Goettingen, Germany) to determine the volume of the recovered dialysate in each vial. Lag time, which is the time it takes for the microdialysate to flow from the membrane to the microvial, was 5.1 minutes and this was taken into account during the data analysis. The flow rate was set to 1.0 $\mu 1 /$ min and vials were replaced at 15 -minute intervals. After each microvial was sampled, it was directly analyzed for glucose, urea, pyruvate and lactate using a microdialysis analyzer (CMA 600, CMA AB, Solna, Sweden) and then frozen to $-20^{\circ} \mathrm{C}$. Within 2 weeks the microvials were thawed and analyzed for insulin concentration by an enzyme-linked immunosorbent assay (Ultrasensitive ELISA, Mercodia AB, Uppsala, Sweden). Four microvials were pooled together to reach the minimum sample volume required $(25 \mu \mathrm{L})$ for the insulin assay. This resulted in one insulin measurement during baseline and one measurement per hour during the oral glucose load.

Capillary blood glucose was collected six times during the experiment; after acclimatization, before giving the glucose load, and every hour for four hours after the oral glucose load. Samples were collected alternately from the index, middle and ring finger of the hand opposite the arm where the microdialysis catheters were inserted. The skin of the fingertip was disinfected (Chlorhexidine, Fresenius Kabi AB, Uppsala, Sweden) and a lancet with 1.8 mm puncture depth (Haemolance Plus, HaeMedic, Ozorków, Poland) was used to puncture the skin. Capillary blood samples were thereafter analyzed directly using a handheld spectrophotometer (Glucose 201+ system, HemoCue AB, Ängelholm, Sweden), which was calibrated according to the manufacturer. Venous blood samples were collected during fasting and two hours after the ingestion of the oral glucose load and directly analyzed by the clinical laboratory at Linköping University Hospital. Samples were taken from the arm opposite the arm where the microdialysis catheters were inserted. After disinfection (Chlorhexidine, Fresenius Kabi AB, Uppsala, Sweden), the median cubital vein was punctured using a butterfly needle (Vacuette ${ }^{\circledR}$, Greiner bio-one, Kremsmünster, Austria) and the sample was directly 
analyzed by the clinical laboratory at Linköping University Hospital. Serum insulin was collected in Serum gel tubes (VWR, Vacutest KIMA, Piove di Sacco, Italy) and analyzed using electrochemiluminescence. Plasma glucose was collected in Citrate/Fluoride/EDTA tubes (Venosafe, Terumo, Västra Frölunda, Sweden) and analyzed using the hexokinase enzymatic method. Insulin resistance and beta-cell function was quantified according to the homeostatic model assessment (HOMA; [fasting insulin $(\mu \mathrm{U} / \mathrm{ml}) \mathrm{x}$ fasting glucose (mmol) / 22.5]) [21].

Skin perfusion was measured every 15 minutes indirectly by the microdialysis urea clearance technique and directly by a Laser Speckle Contrast Imaging (LSCI, PeriCam PSI System, Perimed AB, Järfälla, Sweden). The microdialysis urea clearance technique is based on the rate of diffusion of urea added to the perfusate, which depends on the local blood flow. The method has been described in detail previously [8,9]. With increased blood flow, urea diffuses out of the catheter at a higher rate because of an increased gradient between the inside of the catheter and the interstitium. LSCI uses a divergent laser beam with a wavelength of $785 \mathrm{~nm}$ to illuminate an area of the skin. Skin perfusion is estimated based on the reduction of the local speckle contrast in the reflected image. The technique has previously been described in detail $[3,13]$. Perfusion images were acquired by averaging data from 42 images taken in rapid succession (acquisition time 2 seconds) and then further analyzed by calculating mean perfusion levels in regions of interest using PIMsoft 1.3 (Perimed AB, Järfälla, Sweden). The region of interest size was set to correspond to the area surrounding each catheter $0.4 \mathrm{~cm}$ x $1.4 \mathrm{~cm}$ and was placed over the catheter membrane of the insulin catheter (ROI 1) and the control catheter (ROI 2). An additional control region was measured remote from the catheter (ROI 3). The spatial resolution of the perfusion image was $0.2 \mathrm{~mm} /$ pixel at the used measurement distance of $25 \mathrm{~cm}$. The system was calibrated according to the manufacturer's recommendations.

\section{Data analysis}

Data in the text are presented as means and standard deviations (SD). Blood pressures before and after the experiment, and differences in volume recovery between catheters were compared using paired Student's t test. Changes in skin temperature and insulin concentration in the skin during the 
course of the experiment were analyzed using 1-way analysis of variance for repeated measures, followed by multiple comparisons using Sidak’s correction. Two-way analysis of variance for repeated measures, followed by multiple comparisons using Sidak's correction were used to analyze changes in glucose, lactate, pyruvate, lactate/pyruvate, urea in the skin and skin perfusion as measured using LSCI, (1) between different time points during the experiment and (2) between the control and insulin catheters. The difference in the concentration of urea, lactate and pyruvate between catheters during the period when insulin was given locally was analyzed using paired Student’s t tests.

The alpha level for statistical significance was set to 0.05. All statistical analyses were made with the aid of GraphPad Prism version 5.02 for Windows (GraphPad Software, San Diego California USA, www.graphpad.com).

\section{Results}

All catheters were successfully inserted in the skin and adequate volume recovery was obtained in all microvials (94 $\pm 6 \%$ ). There was no difference in volume recovery between the insulin and control catheters $(\mathrm{p}=0.50)$. Blood pressure did not differ before and after the experiment $(116 / 74$ mmHg and 116/70 mmHg, $\mathrm{p}>0.11$ ). Skin temperature was $31.1 \pm 0.6^{\circ} \mathrm{C}$ after acclimatization and was significantly increased at 2 and 3 hours after oral glucose load (2h: $33.1 \pm 1.0{ }^{\circ} \mathrm{C}$; 3h: $33.2 \pm 0.8$ $\left.{ }^{\circ} \mathrm{C} ; \mathrm{p}=0.007\right)$. Skin temperature did not change in control subjects who did not receive an oral glucose load $(\mathrm{p}>0.29)$. Detailed numeric data can be found in the supplementary files published in conjunction with this article.

\section{Local delivery of insulin}

The changes in concentrations of glucose and insulin in the dialysate are shown in Figure 2. No significant changes in these markers were observed during baseline. During local delivery of insulin, no changes in the concentration of glucose were observed in the insulin catheter $(p=0.97)$ or 
the control catheter $(\mathrm{p}=0.35)$. The changes in the concentration of lactate and pyruvate are shown in Figure 3 and 4, respectively. Lactate and pyruvate concentrations were increased significantly between 15 and 60 minutes of local insulin delivery in the insulin catheter compared with the control catheter (lactate: $\mathrm{p}=0.04$, pyruvate: $\mathrm{p}=0.01$ ). There was no significant change in the lactate/pyruvate ratio after one hour of local delivery of insulin in the insulin catheter $(\mathrm{p}=0.05)$ or in the control catheter ( $\mathrm{p}=0.80$ ), nor was there any difference in lactate/pyruvate ratio between the two catheters ( $\mathrm{p}$ $=0.08)$.

\section{Oral glucose load}

The concentration of glucose in the skin increased during the first hour after intake of an oral glucose load and peaked at 70 minutes after intake of glucose. It remained increased compared with baseline until 3 hours after intake ( $\mathrm{p}=0.01$ ). There was no significant difference in the glucose response between the two catheters (Figure 2). As a result of the effect of local insulin delivery, the lactate concentration at the time of intake of the oral glucose load was significantly higher in the insulin catheter than in the control catheter $(p=0.001)$. Lactate concentrations increased during the first hour and remained elevated until 2 hours (for insulin catheter, $\mathrm{p}<0.001$ ) and 3 hours (for control catheter, $\mathrm{p}<0.001$ ) after oral intake of glucose (Figure 3). Similarly, the concentration of pyruvate in the dialysates was increased significantly until 2 hours after oral intake of glucose $(p<0.002$, Figure 4), with no difference in response between the two catheters, except for a significantly higher lactate concentration in the insulin catheter at the time of intake of glucose $(\mathrm{p}<0.001)$.

The lactate/pyruvate ratio was significantly increased compared to baseline between 3 and 4 hours after intake of glucose ( $\mathrm{p}=0.04$, both catheters), but did not differ significantly between the two catheters (Figure 5).

In control subjects in which no insulin was delivered locally or glucose given orally, no changes in glucose $(p=0.37)$, lactate $(p=0.69)$, pyruvate $(p=0.39)$ or lactate/pyruvate ratio $(p=$ 0.15) were observed during the experiment. 


\section{Skin perfusion}

No significant changes in dialysate concentrations of urea were observed during baseline in any catheter (Figure 6A). After 60 minutes, when the perfusate was switched to insulin, or continued with control solution, a decrease in the urea concentration was observed in both the catheter in which insulin was delivered, as well as in the control catheter. However, the urea concentration was significantly lower during the first 30 minutes of local delivery of insulin in the insulin catheter, compared with control catheter $(\mathrm{p}=0.047)$, indicating a higher local blood flow in the tissue surrounding the insulin catheter.

When glucose was delivered systemically through the oral glucose load, the urea concentration in both catheters continued to decrease at comparable levels, indicating a further increase in skin blood flow during systemic glucose intake independent of local insulin delivery.

In the control subjects who did not receive local insulin at all, no significant change in the urea concentration was observed, although there was a similar, sudden decrease after 60 minutes, and a slight overall decrease over time $(\mathrm{p}=0.43)$. The decrease in urea concentration was more pronounced in the subjects receiving an oral glucose load, compared to the control subjects $(\mathrm{p}=0.01)$.

The skin perfusion around both catheters, as measured using LSCI, increased gradually over time (Figure 6B). The perfusion in the skin around the insulin catheter increased from baseline during local insulin delivery $(\mathrm{p}=0.008)$, whereas no change in perfusion was observed around the control catheter during the same time period $(\mathrm{p}=0.39)$. After the oral glucose load, perfusion around both catheters increased significantly compared to baseline (after $1 \mathrm{~h}$ for the insulin catheter, $\mathrm{p}<0.001$; after $2 \mathrm{~h}$ for the control catheter, $\mathrm{p}=0.001$ ). At the control site remote from the catheters, there was no change in skin perfusion at any time during the experiment $(\mathrm{p}>0.39)$.

In control subjects that did not receive local insulin or an oral glucose load, a slight, nonsignificant increase in skin perfusion, as measured with LSCI, was observed around the catheter, while a decrease from baseline was observed at a remote skin site, which was significant at 1, 3 and 4 hours into the experiment $(\mathrm{p}<0.02)$. After 4 hours, the perfusion was significantly higher around the catheter than at the remote site $(\mathrm{p}<0.001)$. 


\section{Discussion}

To the best of our knowledge, the present study is the first to use microdialysis in the skin to simultaneously demonstrate metabolic and vascular responses after local and endogenous insulin delivery. Also, whereas other studies have detected changes in insulin levels in muscle and adipose tissue after an oral glucose load [32], this study is the first to detect a change in insulin levels in the skin. The main finding of this study is that the time course of the effects of hyperinsulinemia on glucose uptake in the skin differs markedly depending on whether insulin is delivered directly to the interstitium via microdialysis, or delivered via a systemic route (after oral glucose intake), and that local delivery of insulin to the skin results in rapid vasodilatation, paralleled by an increased glucose uptake.

Microdialysis has been used to study metabolic and vasoactive actions of insulin in brain [36], skeletal muscle and subcutaneous adipose tissue [28]. Only a few studies have however used microdialysis to study the effects of insulin in skin. Jansson et al. studied changes in lactate and blood flow in the skin during an oral glucose tolerance test and found that the skin releases lactate during fasting but that the net release of lactate from the skin is not increased after OGTT, and that no increase in blood flow occurs during OGTT as measured with ${ }^{133}$ Xe clearance [16].

In the current study, we found an immediate, within 15 min, increase in tissue lactate and pyruvate during local insulin delivery. This indicates a rapid, increased glucose uptake in the skin as a result of increased interstitial insulin levels. Similarly, when Chiu et al. administered insulin directly into skeletal muscle they detected a rapid increase in glucose disposal [4]. They concluded that once insulin arrives in the interstitial space, all remaining steps for insulin to mediate glucose uptake are not temporally limiting, i.e. the rate limiting trans-endothelial transport is bypassed. Our results are consistent with these findings. Interestingly, in our results the interstitial glucose concentration did not decrease during local delivery of insulin. We assume that the reason why we did not observe this is because of a high transport capacity for glucose from the vasculature to the interstitium compared to 
its metabolism, even when glucose uptake is increased as a result of increased levels of insulin [19]. This may be specific for the dermal interstitium, as a previous study has shown a decreasing interstitial glucose concentration after local insulin delivery to skeletal muscle [27].

After an oral glucose load, the peak in the blood glucose concentration is usually observed between 30 and 60 min after glucose intake [18,24]. In this study, we found a peak in glucose concentration in the skin after 68 minutes, while interstitial lactate and pyruvate peaked somewhat later, at 83 minutes. The peak in interstitial insulin was observed between 68 and 128 minutes after oral glucose intake. Because of the limited temporal resolution it is difficult to determine a more exact time point for the peak in interstitial insulin, but it is likely to occur after the peak in interstitial glucose. This finding is in agreement with the idea that the endothelium limits the availability of insulin in the interstitial space of the skin.

The increased levels of lactate and pyruvate in the skin after the oral glucose load were rather similar for the insulin and control catheters. This indicates that the increase in glucose uptake with locally delivered insulin was modest compared with the increase in glucose uptake after systemic hyperglycemia.

It has been known for many years that insulin has direct vasoactive properties [1]. It is now generally believed that insulin to some extent regulates its own delivery, and that the delivery of glucose to muscle tissue is mediated through direct effects on the vascular endothelium. Many studies have used systemic clamps with supraphysiological insulin concentrations to investigate the vasoactive effects of insulin on muscle microcirculation, and these have generally showed vasodilating effects and increased limb blood flow [20,26]. Other studies have delivered insulin locally in the skin, and have found an increase in skin blood flow independent of any systemic effect $[14,34]$. Whether physiological levels of insulin increase total limb blood flow has, however, been more controversial, and insulin is now instead considered to redirect blood flow from non-nutritive to the nutritive vascular bed, even at physiological concentrations, a process known as capillary recruitment [5,37]. The majority of studies investigating the vascular effects of insulin have been using skeletal muscle tissue as the organ of interest. It has become clear, however, that changes in the vascular actions of insulin in skeletal muscle are paralleled by changes in cutaneous microcirculation 
$[22,34]$. The skin has therefore emerged as a popular surrogate organ to study these effects, because it is easily accessible for measurement of changes in skin microcirculation using minimally-invasive techniques.

The findings in this study add to the evidence that insulin causes vasodilatation in the microcirculation in the skin. More urea was cleared from the catheter in which insulin was delivered locally, and an increase in skin perfusion was observed with LSCI, indicating that microvascular blood flow in the skin was increased. It may be expected that high concentrations of insulin delivered to the interstitium will cause endothelium-dependent vasodilatation, considering the known vasoactive properties of insulin. It should however be noted that the direction of insulin transport to the endothelium in this case is reversed from the normal route, as the normal active transendothelial transport mechanisms are bypassed. On the other hand, insertion of the catheter will likely cause a disruption of the local microvascular structure, causing an increased availability of insulin to the endothelium that may have a direct effect on the microvasculature through vasodilatation and capillary recruitment.

After the oral glucose load, an increased urea clearance was observed in both microdialysis catheters. The insertion of microdialysis catheters in the skin is known to cause a local rise in inflammatory cytokines that is sustained for 48h [35]. This inflammatory cascade may be responsible for the slight increase in blood flow near the observed in the control catheters and in the subjects who did not receive local insulin or an oral glucose load. However, the change in skin perfusion was significantly more pronounced in the subjects receiving an oral glucose load, than in the control subjects. This suggests that tissue blood flow is increased secondary to systemic hyperglycemia, and that this effect is independent of local insulin delivery. An increased skin blood flow near the catheters was also observed with LSCI in the subjects receiving oral glucose (and not in the control subjects). However, no change in blood flow was observed at a control site distant to the catheters. The absence of blood flow changes in unprovoked skin indicates that a systemic glucose load does not increase total skin blood flow, despite increased insulin levels and an increased glucose metabolism in the skin. The question why LSCI fails to show an increase in perfusion after an oral glucose load, at a skin site remote from the catheter, is difficult to answer, but one explanation may be the difference in 
measurement volume between the techniques. Because the catheters are located near the subdermis, it is likely that the effects on urea clearance are related to changes in subdermal blood flow, whereas LSCI is sensitive to changes in perfusion in the superficial dermis [25]. One could therefore speculate that LSCI measures too superficially to detect any microvascular effects in the skin after a systemic glucose load. Finally, it should be noted that there was a significant decrease in skin perfusion during the experiment in the control subjects, whereas the perfusion was unchanged in the subjects receiving the oral glucose load. Thus, the sensitivity of LSCI may be too low or our study may have been underpowered to detect any significant changes in skin perfusion after the oral glucose load.

\section{Limitations}

A limitation of the study was that we do not know the actual concentration of insulin delivered to the skin by the microdialysis catheter, as we did not analyze the concentration of insulin aspart in the dialysate. However, the perfusate concentration was six orders of magnitude higher than fasting insulin levels ( $\sim 10 \mathrm{mU} / \mathrm{L}$ ), so it is very likely that supraphysiological levels are delivered to the interstitium, even with an expected low reverse recovery of 10\% [15]. There has been limited research on how much insulin traverses the catheter membrane, but insulin is known to have the tendency to adhere to the membrane of the tubing. We wanted to start out on the safe side and deliver a high dose, to ensure that insulin specific effects in the tissue would be detectable. Future studies will focus on the transport characteristics of insulin over the membrane in vitro and in vivo, and will assess potential insulin adherence to the membrane.

\section{Conclusion}

Intracutaneous microdialysis makes it possible to study the metabolic and microvascular effects of insulin in the skin with a high temporal resolution. By using the urea clearance method, changes in microvascular blood flow can be measured in the same sampling volume as where insulin is delivered, and where glucose uptake takes place. Local delivery of supraphysiological 
concentrations of insulin caused an immediate (within 15 minutes) increase in microvascular blood flow, as measured using two different techniques. This increase in blood flow was paralleled by simultaneous increases in interstitial pyruvate and lactate levels, indicating increased glucose uptake. These results provide further evidence that insulin in high concentrations rapidly increases microvascular blood flow in the skin. After an oral glucose load, the maximum interstitial glucose level was observed at 68 minutes, while maximum pyruvate and lactate levels were observed at 83 minutes. A peak in interstitial insulin was observed between 60 and 120 minutes after oral glucose intake. Thus, the availability of insulin and the resulting metabolic effects in the interstitium of the skin are delayed after a systemic glucose load compared to when insulin is delivered locally, suggesting a limiting effect of the microvasculature.

We observed an increase in urea clearance from the catheters, compared to control subjects who did not receive an oral glucose load, which suggests an increase in blood flow in the skin mediated by systemic release of insulin. However, the fact that we did not observe any changes in skin perfusion using LSCI indicates that an oral glucose load does not alter perfusion in the superficial microcirculation much. . Further studies are needed to investigate whether vasodilatory effects of insulin in the skin at physiological concentrations are relevant for the delivery of insulin and glucose to the interstitium.

\section{Acknowledgements}

We thank Florence Sjögren for her help with the chemical analyses of interstitial insulin in the dialysate samples.

\section{Figure legends}

Figure 1. Experimental protocol and setup. 
Figure 2. Changes in the concentration of glucose (circles) and insulin (diamonds) in the skin during continuous intradermal delivery of insulin (gray markers) or a control substance (black markers), and after oral intake of a $75 \mathrm{~g}$ glucose load, in healthy subjects $(\mathrm{N}=8)$. A separate group of 6 subjects (open circles) did not receive intradermal insulin or oral glucose load and served as controls.

Figure 3. Changes in the concentration of lactate in the skin during continuous intradermal delivery of insulin (gray markers) or a control substance (black markers), and after oral intake of a 75 g glucose load, in healthy volunteers $(\mathrm{N}=8)$. A separate group of 6 subjects (open circles) did not receive intradermal insulin or oral glucose load and served as controls. * indicates a significant difference between the insulin catheter and control catheter.

Figure 4. Changes in the concentration of pyruvate in the skin during continuous intradermal delivery of insulin (gray markers) or a control substance (black markers), and after oral intake of a $75 \mathrm{~g}$ glucose load, in healthy volunteers. A separate group of 6 subjects (open circles) did not receive intradermal insulin or oral glucose load and served as controls. * indicates a significant difference between the insulin catheter and control catheter.

Figure 5. Changes in the lactate/pyruvate ratio in the skin during continuous intradermal delivery of insulin (gray markers) or a control substance (black markers), and after oral intake of a 75 g glucose load, in healthy volunteers. A separate group of 6 subjects (open circles) did not receive intradermal insulin or oral glucose load and served as controls.

Figure 6. Skin perfusion during continuous intradermal delivery of insulin (grey markers) or a control substance (black markers), and after oral intake of a 75 g glucose load, in healthy volunteers. A. Intradermal blood flow around the catheters as measured using the urea clearance technique. B. Skin perfusion around the microdialysis catheters as measured using laser speckle contrast imaging. The skin perfusion in an area remote from the catheters is shown in white markers. A separate group of 6 subjects (open markers) did not receive intradermal insulin or oral glucose load and served as controls. * indicates a significant difference between the insulin catheter and control catheter. Special markers in figure $\mathrm{B}(\star, \star$ and $\otimes)$ indicate a significant change from baseline.

\section{References}

1. Baron AD. Hemodynamic actions of insulin. Am J Physiol 267: E187-202, 1994. 
2. Barrett EJ, Eggleston EM, Inyard AC, Wang H, Li G, Chai W, Liu Z. The vascular actions of insulin control its delivery to muscle and regulate the rate-limiting step in skeletal muscle insulin action. Diabetologia 52: 752-764, 2009.

3. Briers JD. Laser Doppler, speckle and related techniques for blood perfusion mapping and imaging. Physiol Meas 22: R35-66, 2001.

4. Chiu JD, Richey JM, Harrison LN, Zuniga E, Kolka CM, Kirkman E, Ellmerer M, Bergman RN. Direct administration of insulin into skeletal muscle reveals that the transport of insulin across the capillary endothelium limits the time course of insulin to activate glucose disposal. Diabetes 57: 828835, 2008.

5. Clark MG. Impaired microvascular perfusion: a consequence of vascular dysfunction and a potential cause of insulin resistance in muscle. Am J Physiol Endocrinol Metab 295: E732-750, 2008.

6. Clark MG, Wallis MG, Barrett EJ, Vincent MA, Richards SM, Clerk LH, Rattigan S. Blood flow and muscle metabolism: a focus on insulin action. Am J Physiol Endocrinol Metab 284: E241-258, 2003.

7. de Jongh RT, Serne EH, RG IJ, de Vries G, Stehouwer CD. Impaired microvascular function in obesity: implications for obesity-associated microangiopathy, hypertension, and insulin resistance. Circulation 109: 2529-2535, 2004.

8. Farnebo S, Samuelsson A, Henriksson J, Karlander LE, Sjoberg F. Urea clearance: a new method to register local changes in blood flow in rat skeletal muscle based on microdialysis. Clin Physiol Funct Imaging 30: 57-63, 2010.

9. Farnebo S, Zettersten EK, Samuelsson A, Tesselaar E, Sjoberg F. Assessment of blood flow changes in human skin by microdialysis urea clearance. Microcirculation 18: 198-204, 2011.

10. Folkesson KT, Samuelsson A, Tesselaar E, Dahlstrom B, Sjoberg F. A human vascular model based on microdialysis for the assessment of the vasoconstrictive dose-response effects of norepinephrine and vasopressin in skin. Microcirculation 19: 352-359, 2012.

11. Hickner RC, Ungerstedt U, Henriksson J. Regulation of skeletal muscle blood flow during acute insulin-induced hypoglycemia in the rat. Diabetes 43: 1340-1344, 1994.

12. Holowatz LA, Thompson-Torgerson CS, Kenney WL. The human cutaneous circulation as a model of generalized microvascular function. J Appl Physiol 105: 370-372, 2008.

13. Iredahl F, Lofberg A, Sjoberg F, Farnebo S, Tesselaar E. Non-Invasive Measurement of Skin Microvascular Response during Pharmacological and Physiological Provocations. PLoS one 10: e0133760, 2015.

14. Iredahl F, Tesselaar E, Sarker S, Farnebo S, Sjoberg F. The Microvascular Response to Transdermal Iontophoresis of Insulin is Mediated by Nitric Oxide. Microcirculation 20: 717-723, 2013.

15. Jansson PA, Fowelin JP, von Schenck HP, Smith UP, Lonnroth PN. Measurement by microdialysis of the insulin concentration in subcutaneous interstitial fluid. Importance of the endothelial barrier for insulin. Diabetes 42: 1469-1473, 1993.

16. Jansson PA, Krogstad AL, Lonnroth P. Microdialysis measurements in skin: evidence for significant lactate release in healthy humans. Am J Physiol 271: E138-142, 1996. 
17. Keske MA, Clerk LH, Price WJ, Jahn LA, Barrett EJ. Obesity blunts microvascular recruitment in human forearm muscle after a mixed meal. Diabetes care 32: 1672-1677, 2009.

18. Kramer CK, Vuksan V, Choi H, Zinman B, Retnakaran R. Emerging parameters of the insulin and glucose response on the oral glucose tolerance test: reproducibility and implications for glucose homeostasis in individuals with and without diabetes. Diabetes Res Clin Pract 105: 88-95, 2014.

19. Kusters YH, Barrett EJ. Muscle's microvasculature's structural and functional specializations facilitate muscle metabolism. Am J Physiol Endocrinol Metab 15;310: E379-387, 2016.

20. Laakso M, Edelman SV, Brechtel G, Baron AD. Decreased effect of insulin to stimulate skeletal muscle blood flow in obese man. A novel mechanism for insulin resistance. J Clin Invest 85: 18441852, 1990.

21. Matthews DR, Hosker JP, Rudenski AS, Naylor BA, Treacher DF, Turner RC. Homeostasis model assessment: insulin resistance and beta-cell function from fasting plasma glucose and insulin concentrations in man. Diabetologia 28: 412-419, 1985.

22. Meijer RI, De Boer MP, Groen MR, Eringa EC, Rattigan S, Barrett EJ, Smulders YM, Serne EH. Insulin-induced microvascular recruitment in skin and muscle are related and both are associated with whole-body glucose uptake. Microcirculation 19: 494-500, 2012.

23. Montero D, Walther G, Perez-Martin A, Santamaria C, Roche E, Mercier C, Vinet A. Decreased microvascular myogenic response to insulin in severely obese adolescents. Clin Hemorheol Microcirc 57: 23-32, 2014.

24. Nolfe G, Spreghini MR, Sforza RW, Morino G, Manco M. Beyond the morphology of the glucose curve following an oral glucose tolerance test in obese youth. Eur J Endocrinol 166: 107-114, 2012.

25. O'Doherty J, McNamara P, Clancy NT, Enfield JG, Leahy MJ. Comparison of instruments for investigation of microcirculatory blood flow and red blood cell concentration. J Biomed Opt 14: 034025, 2009.

26. Raitakari M, Knuuti MJ, Ruotsalainen U, Laine H, Makea P, Teras M, Sipila H, Niskanen T, Raitakari OT, Iida H, Harkonen R, Wegelius U, Yki-Jarvinen H, Nuutila P, et al. Insulin increases blood volume in human skeletal muscle: studies using [15O]CO and positron emission tomography. Am J Physiol 269: E1000-1005, 1995.

27. Rosdahl H, Hamrin K, Ungerstedt U, Henriksson J. A microdialysis method for the in situ investigation of the action of large peptide molecules in human skeletal muscle: detection of local metabolic effects of insulin. Int J Biol Macromo 28: 69-73, 2000.

28. Rosdahl H, Lind L, Millgard J, Lithell H, Ungerstedt U, Henriksson J. Effect of physiological hyperinsulinemia on blood flow and interstitial glucose concentration in human skeletal muscle and adipose tissue studied by microdialysis. Diabetes 47: 1296-1301, 1998.

29. Rossi M, Maurizio S, Carpi A. Skin blood flowmotion response to insulin iontophoresis in normal subjects. Microvasc Res 70: 17-22, 2005.

30. Rossi M, Santoro G, Ricco R, Pentimone F, Carpi A. Effect of chronic aerobic exercise on cutaneous microcirculatory flow response to insulin iontophoresis and to ischemia in elderly males. Int J Sports Med 26: 558-562, 2005. 
31. Samuelsson A, Farnebo S, Magnusson B, Anderson C, Tesselaar E, Zettersten E, Sjoberg F. Implications for burn shock resuscitation of a new in vivo human vascular microdosing technique (microdialysis) for dermal administration of noradrenaline. Burns 38: 975-983, 2012.

32. Sandqvist M, Strindberg L, Schmelz M, Lonnroth P, Jansson PA. Impaired delivery of insulin to adipose tissue and skeletal muscle in obese women with postprandial hyperglycemia. J Clin Endocrinol Metab 96: E1320-1324, 2011.

33. Serne EH, Gans RO, ter Maaten JC, ter Wee PM, Donker AJ, Stehouwer CD. Capillary recruitment is impaired in essential hypertension and relates to insulin's metabolic and vascular actions. Cardiovasc Res 49: 161-168, 2001.

34. Serne EH, RG IJ, Gans RO, Nijveldt R, De Vries G, Evertz R, Donker AJ, Stehouwer CD. Direct evidence for insulin-induced capillary recruitment in skin of healthy subjects during physiological hyperinsulinemia. Diabetes 51: 1515-1522, 2002.

35. Sjogren F, Anderson C. Sterile trauma to normal human dermis invariably induces IL1beta, IL6 and IL8 in an innate response to "danger". Acta Derm Venereol 89: 459-465, 2009.

36. Vespa P, Boonyaputthikul R, McArthur DL, Miller C, Etchepare M, Bergsneider M, Glenn T, Martin N, Hovda D. Intensive insulin therapy reduces microdialysis glucose values without altering glucose utilization or improving the lactate/pyruvate ratio after traumatic brain injury. Crit Care Med 34: 850-856, 2006.

37. Vincent MA, Clerk LH, Lindner JR, Klibanov AL, Clark MG, Rattigan S, Barrett EJ. Microvascular recruitment is an early insulin effect that regulates skeletal muscle glucose uptake in vivo. Diabetes 53: 1418-1423, 2004.

38. Vincent MA, Clerk LH, Lindner JR, Price WJ, Jahn LA, Leong-Poi H, Barrett EJ. Mixed meal and light exercise each recruit muscle capillaries in healthy humans. Am J Physiol Endocrinol Metab 290: E1191-1197, 2006. 
Table 1. Demographics of the subjects enrolled in the study. Data presented in I

\begin{tabular}{|c|c|c|c|}
\hline & & Experimental subjects & Control subjects \\
\hline $\mathrm{N}$ & & 8 & 6 \\
\hline Sex & (female/male) & $4 / 4$ & $3 / 3$ \\
\hline Age & (years) & $23.0(6.9)$ & $23.0(0.6)$ \\
\hline BMI & $\left(\mathrm{kg} / \mathrm{m}^{2}\right)$ & $22.2(1.8)$ & $23.2(2.5)$ \\
\hline Blood pressure & (mmHg) & & \\
\hline Before experimen & & 116/73 (11/9) & $116 / 74(14 / 10)$ \\
\hline After experiment & & $117 / 68(8 / 5)$ & $115 / 72(10 / 10)$ \\
\hline Serum insulin & $(\mathrm{mU} / \mathrm{L})$ & & \\
\hline Fasting & & $7.2(2.1)$ & $8.5(3.0)$ \\
\hline 2 hours OGTT & & $38.1(24.9)$ & $5.3(0.8)$ \\
\hline Capillary glucose & $(\mathrm{mmol} / \mathrm{L})$ & & \\
\hline Fasting & & $5.3(0.5)$ & $4.6(0.2)$ \\
\hline 1 hour OGTT & & $10(1.2)$ & $(4.6(0.2))$ \\
\hline 2 hours OGTT & & $6.7(1.0)$ & $(4.6(0.2))$ \\
\hline 3 hours OGTT & & $5.9(1.3)$ & $(4.3(0.2))$ \\
\hline 4 hours OGTT & & $4.3(0.7)$ & $(4.3(0.3))$ \\
\hline HOMA IR & ${ }^{\circ} \mathrm{C}$ & $1.72(0.6)$ & $1.86(0.7)$ \\
\hline Skin temperature & () & & \\
\hline Fasting & & $31.5(1.1)$ & $31.3(1.3)$ \\
\hline 1 hour OGTT & & $32.5(1.2)$ & $(31.3(1.0))$ \\
\hline 2 hours OGTT & & $33.1(1.0)$ & $(31.2(1.0))$ \\
\hline 3 hours OGTT & & $33.2(0.8)$ & $(31.3(1.2))$ \\
\hline 4 hours OGTT & & $32.9(0.7)$ & $(31.0(1.6))$ \\
\hline
\end{tabular}


mean and standard deviation (SD). 

glucose (insulin catheter)

glucose (control catheter)

insulin (control catheter)

glucose (control subjects)

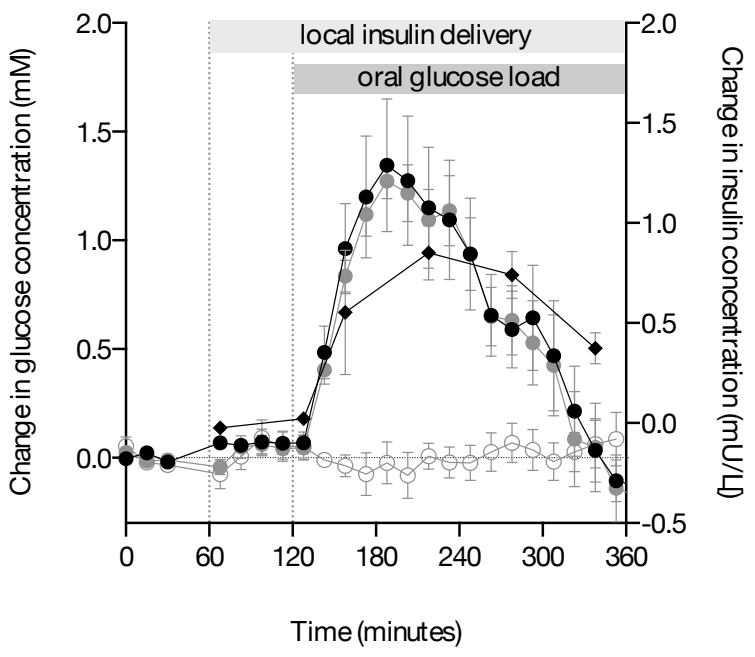


- - insulin catheter

- control catheter

- control subjects

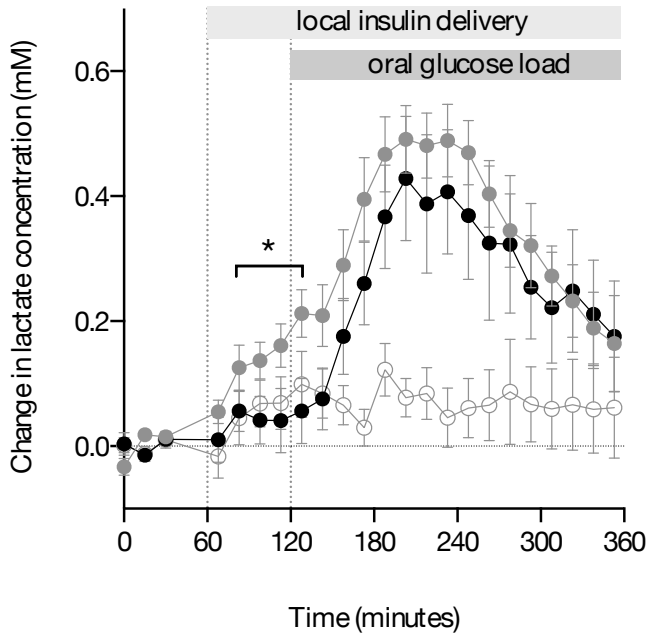


- insulin catheter

- control catheter

$-\quad$ control subjects

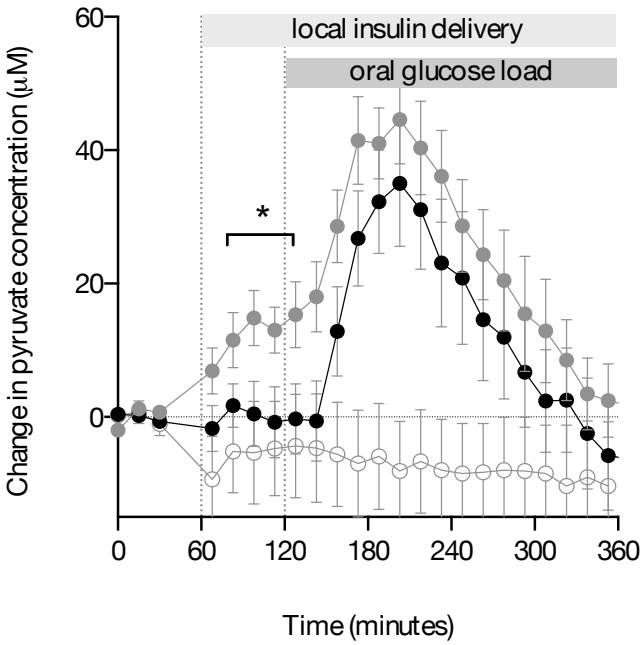




\section{- insulin catheter \\ - control catheter \\ - control subjects}

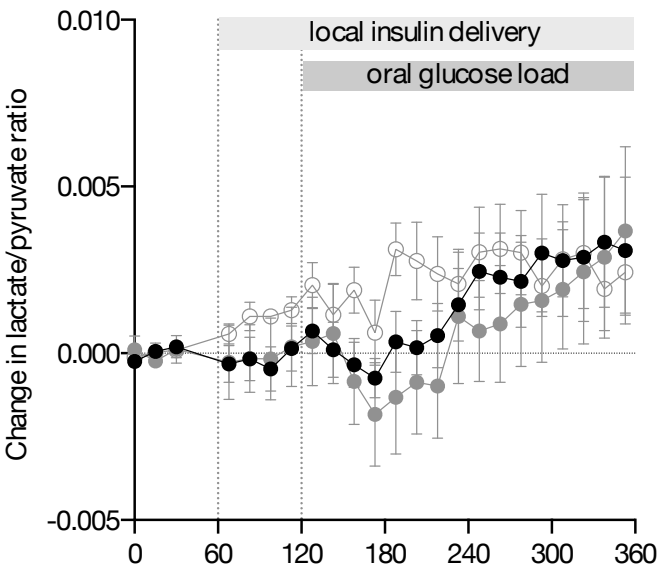

Time (minutes) 
A

B

- - insulin catheter

$\rightarrow \quad$ control catheter

- control subjects

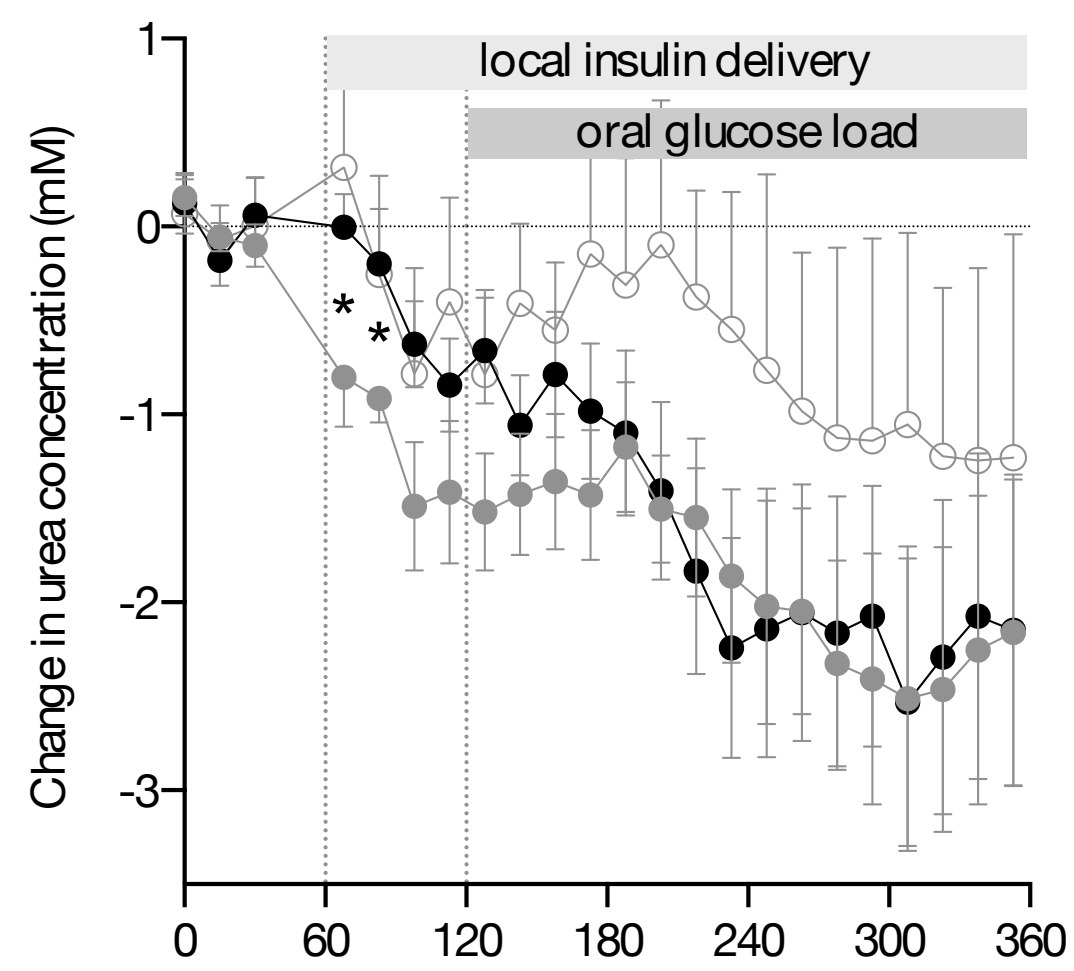

Time (minutes)
- insulin catheter

- control catheter

- remote skin site

$-\quad$ control subjects (catheter)

$\square$ control subjects (remote skin site)

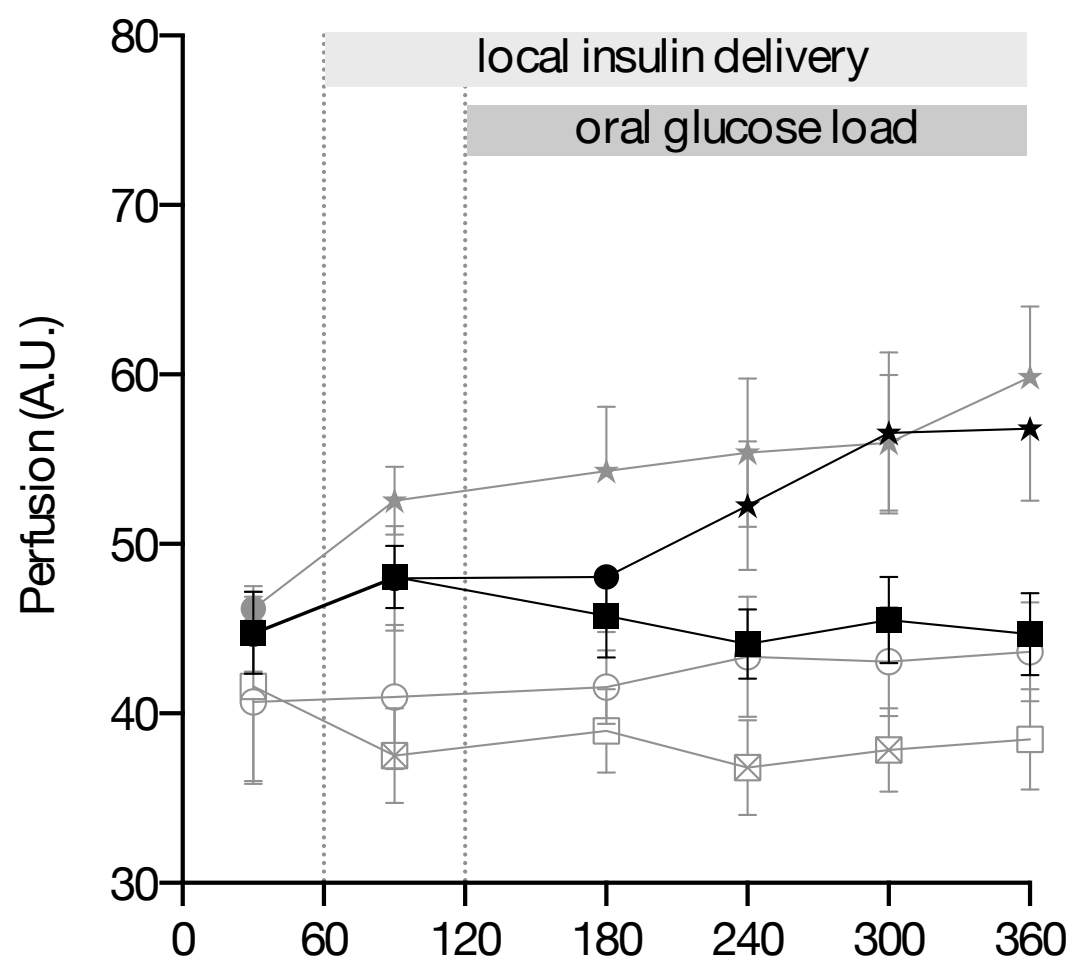

Time (minutes) 